\title{
Parametric design of shells in reinforced concrete: a case study of the Los Manantiales
}

\author{
Camilla Ribeiro do Rozário \\ Universidade Estadual de Campinas | Brazil | camillarozario@gmail.com.br \\ Felipe da Silva Tavares \\ Universidade Federal da Bahia | Brazil I felipe.tavares@ufba.br
}

\begin{abstract}
In this article it is proposed a study of reinforced concrete shells modeling, taking as case study the shell roof building designed by Candela in Xochimilco, Los Manantiales. The approach for this study is related to the comprehension of both project methodologies: the one before the use of the new emerging technologies and the one after. The objective of this study is to measure the structural efficiency of shells designed by both approaches and, simultaneously, how the emergence of new computational technologies and software's could influence in the design process in the civil engineering and architecture's design.
\end{abstract}

Keywords: Parametric design; Form finding; Shells; Reinforced concrete.

\section{INTRODUÇÃO}

O uso das tecnologias CAD (Computer Aided Design) tem um reflexo direto no processo projetual de Arquitetura e no seu ensino, uma vez que podem incorporar simulações digitais de fenômenos físicos que ocorrem nos sistemas prediais. Sabe-se que as simulações digitais já são, há algum tempo, fundamentais no processo de projeto dos sistemas das edificações. Desta maneira, a compreensão sobre as possibilidades trazidas por tais ferramentas tornase imprescindível para uma adequada proposição de novos projetos e concepções estruturais, sobretudo em estruturas de comportamento espacial, como é o caso das cascas em concreto armado.

Neste artigo, propõe-se um processo de projeto paramétrico de estruturas de cascas em concreto armado com função de cobertura, tomando como caso de estudo - Los Manantiales de Félix Candela e, também, as avaliações de outra opção de geometria semelhante, obtida através do método form-finding. Com relação a esta última, pode-se dizer que as cascas obtidas por formfinding são estruturas funiculares que se destacam especialmente pela sua alta eficiência estrutural, por estarem submetidas majoritariamente à compressão simples.

De acordo com Tomas e Martí-Montrull (2010) estas tipologias de estruturas não dependem apenas das espessuras estruturais para se tornarem estáveis, mas de seu formato global também. Foi através dessa ideia que diversos arquitetos e engenheiros, tais como Gaudi, Isler e Candela projetaram e construíram formas de extrema leveza que possuíam um aspecto elegante ao mesmo tempo em que apresentavam uma economia de materiais.

Considerando-se que o projeto estrutural de cascas é relativamente complexo quando não há a possibilidade de se utilizar ferramentas computacionais, pode-se concluir que provavelmente por isso justifica-se o fato destes projetistas terem projetado utilizando modelos físicos em escala reduzida para a obtenção da forma. Neste sentido, os atuais softwares CAD paramétricos se apresentam como uma ferramenta com potencial de tornar a concepção de tais formas mais acessível aos projetistas, uma vez que estas lidam com a complexidade da modelagem mais adequadamente e ampliam, consequentemente, as capacidades projetuais dos profissionais e estudantes de arquitetura.

Procurou-se, assim, compreender a concepção destas cascas, tanto em relação a um momento prévio ao uso destas tecnologias digitais quanto em relação a um momento posterior. Para isto foi utilizado o método de obtenção de formas funiculares, form-finding, para analisar a tipologia da casca de Los Manantiales e verificar seu comportamento estrutural frente aos esforços, deslocamentos por deformação e à flambagem.

Ao aplicar o método de form-finding digital sobre a projeção da forma curva de Los Manantiales, verificou-se que a forma resultante não corresponde à forma original projetada por Félix Candela, o que levou a uma discussão sobre como esta foi realmente concebida. Surgiu então a indagação: As cascas concebidas por Candela são efetivamente funiculares como se espera que as cascas mais eficientes sejam?

Na concepção de estruturas de cascas ou gridshells, uma das maneiras de se conceber a forma global é utilizando algum método form-finding, analógico ou digital, ou por outro lado, utilizar a geração deliberada da geometria, sem considerar de início a relação intrínseca entre forma geométrica deste tipo de estrutura e o equilíbrio gravitacional da mesma (Dimcic,2011; Rippmann,2016). Em ambos os casos, a forma gerada necessita ser avaliada estruturalmente e verificada de acordo com os preceitos de aceitabilidade estrutural estipulados pelas normas de projeto estrutural vigentes.

Nesta direção, compreender como Candela concebeu e viabilizou a construção de suas formas torna-se um 
importante fator para fundamentar esta discussão. As formas por ele produzidas não foram desenvolvidas através de ferramentas computacionais, mas sim através de um conhecimento de engenharia em conjunto com a necessidade de buscar economia no projeto e eficiência no consumo dos materiais (Garlock et al.,2008). Apesar dos poucos relatos sobre o processo projetual utilizado por Candela para a concepção de suas formas, torna-se evidente que a produção feita por ele remete a em um processo empírico ou experimental.

A trajetória de Candela esteve embasada especialmente em questionamentos referentes aos cálculos estruturais tradicionais da época (Candela,1962). As críticas realizadas por ele em relação aos métodos de cálculo estrutural tradicionais o levaram a buscar caminhos alternativos à época, propondo algumas cascas através de métodos geométricos de determinação da forma. A produção de Candela, neste âmbito, demonstrou possuir um aspecto experimental e, por isso, o seu processo de produção não foi sempre bem-sucedido, uma vez que grande parte de sua produção se embasou em métodos empíricos (Garlock et al.,2008). Como exemplo, pode-se mencionar a casca do projeto de Candela para Lomas de Cuernavaca que entrou em colapso durante o processo de construção e teve que ser reconstruída com uma espessura maior (Tomas e Martí-Montrull,2010). Uma possível hipótese para explicar as falhas ocorridas neste colapso seria a menor precisão na definição da forma e na compreensão do comportamento estrutural modelado através de cálculos matemáticos manuais simplificados.

\section{METODOLOGIA}

Tendo em vista a eficiência de tais cascas, este estudo fundamentou-se na análise de duas cascas: uma gerada por Candela e outra, semelhante a esta primeira, gerada a partir da metodologia do form-finding digital.

Dentre as diversas técnicas de form-finding utilizadas na obtenção de formas tridimensionais funiculares, foi aqui utilizada a técnica denominada como hanging-chain (Dimcic,2011; Rippmann,2016). Esta técnica foi utilizada por alguns projetistas que conceberam estruturas funiculares, em épocas de pouco desenvolvimento computacional, utilizando-se assim modelos físicos em escala reduzida, compostos por uma rede de correntes que simulam o funcionamento de uma rede de cabos funiculares. Sabe-se que o funcionamento do cabo funicular é o oposto dos arcos funiculares, os quais, por sua vez, são uma versão bidimensional das cascas funiculares.

Além do form-finding digital, com o desenvolvimento do método dos elementos finitos em ambientes computacionais, os modelos estruturais ficaram mais sofisticados e precisos na simulação do comportamento estrutural e obtenção dos esforços de projeto.

Além da análise sobre os resultados da simulação estrutural realizada sobre a forma obtida pelo form-finding digital, foi assim também feito sobre a forma original de Los Manantiales, obtida pela abordagem geométrica por Candela. Para realizar a modelagem geométrica digital da forma original foram utilizadas as dimensões e proporções do edifício encontradas em imagens e textos contidos na literatura (Faber, 1963).
Nesta seção, serão detalhadas as técnicas utilizadas para a obtenção das formas funiculares de cascas, a modelagem paramétrica da geometria original e a verificação da aceitabilidade de deslocamentos e esforços estruturais segundo a norma brasileira para projeto de estruturas de concreto armado NBR 6118 (2014).

\section{FORM-FINDING DIGITAL}

O conceito de form-finding tem como base a obtenção de uma determinada forma a partir da distribuição de cargas permanentes sobre esta. A estrutura resultante obtida por este processo tem uma conformação característica que garante o seu equilíbrio gravitacional autonomamente e, assim, tende a apresentar menores deslocamentos e uma predominância de esforços de compressão simples.

Neste estudo foi utilizado a técnica hanging-chain, implementando-a no ambiente do programa Rhinoceros, utilizando o plugin Grasshopper e seus add-ons Weaverbird e Karamba. Numericamente, este método é formulado através da técnica force density method (Dimcic,2011).

Em termos práticos, os dados de entrada deste método são: um mesh, que consiste na projeção horizontal da casca que será obtida; a fixação deliberada do deslocamento de alguns nós deste mesh; e a definição de uma altura máxima de deslocamentos dos nós que estão livres para se movimentar durante o processo numérico. $A$ movimentação destes nós será dada pela aplicação de forças unitárias com direção vertical, representando a ação da gravidade, onde as arestas do mesh em questão funcionam como molas unidirecionais.

Para aplicar esta técnica do form-finding considerando um mesh sobre a planta do Los Manantiales, foram modeladas uma sequência de curvas NURBS sobre o contorno da planta deste edifício (Faber, 1963), ilustrado pela Figura 1.

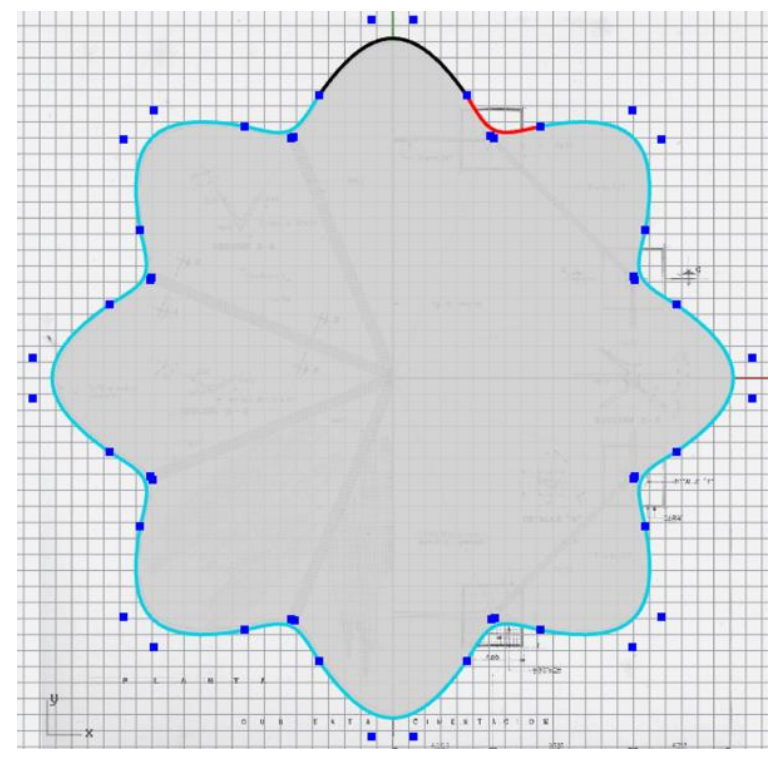

Figura 1: Curvas no contorno da planta do Los Manantiales. Fonte: Autores.

Nesta figura, observa-se em preto as curvas que contornam as regiões de abertura de circulação espacial 


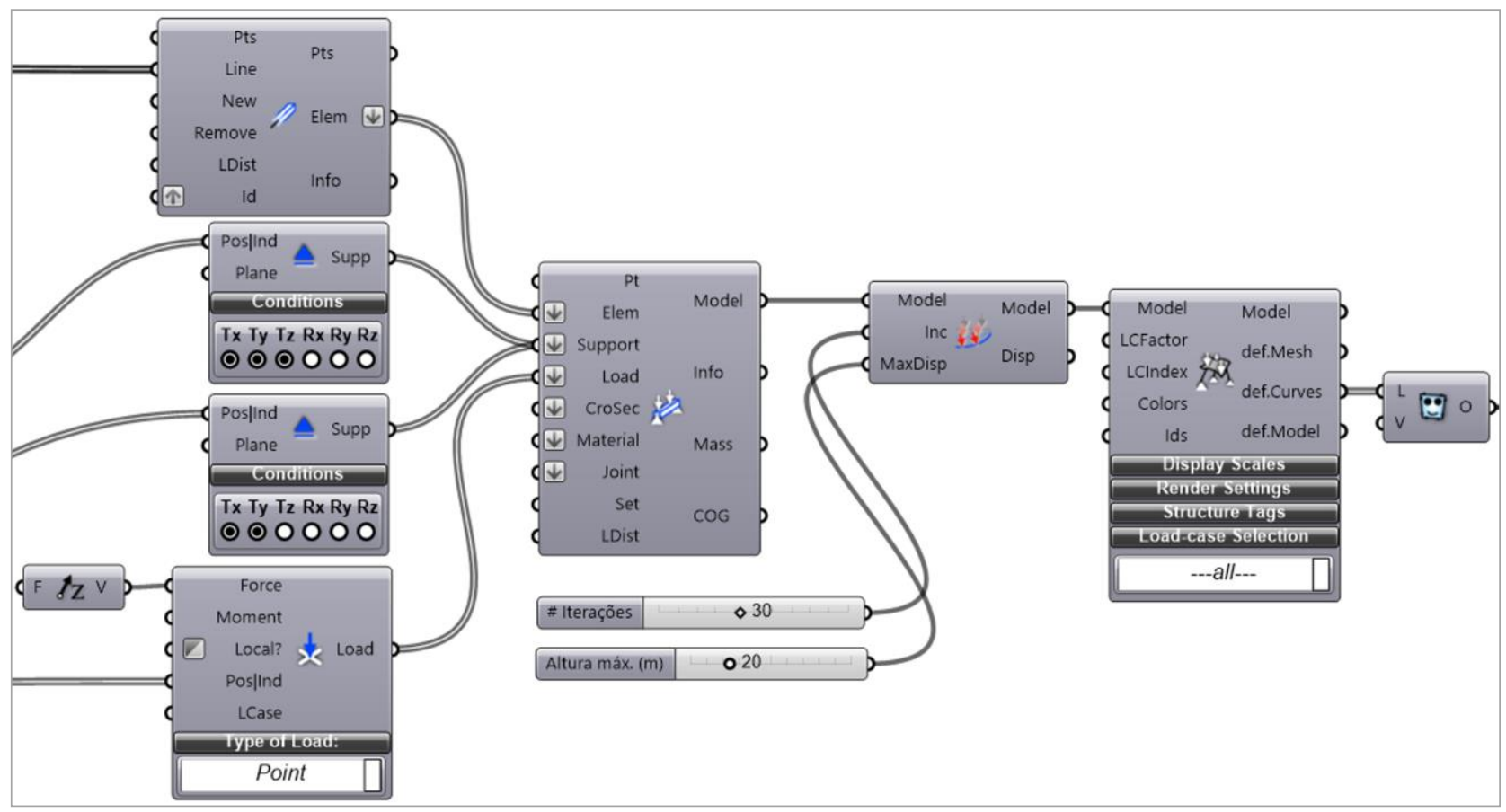

Figura 2: Código do form-finding pelo Karamba e reconstrução do mesh a partir das linhas utilizando o Weaverbird. Fonte: Autores.

da edificação e, em vermelho, as curvas que estão na região dos apoios da estrutura do projeto original. Os pontos em azul são os pontos de controle das curvas.

A superfície conformada por este conjunto de curvas foi então convertida em um mesh de face triangular para se obter as curvaturas melhor representadas. As arestas deste mesh triangular, extraídas como segmentos de linha, foram convertidas em elementos estruturais de barras através do componente do Karamba Line-to-Beam. Ainda, os pontos presentes nas regiões de apoio estrutural no projeto original foram definidos como apoios, impedindo os deslocamentos translacionais nos três eixos. Os pontos presentes nas arestas externas do mesh (naked edges) foram definidos como fixos nos eixos horizontais, permitindo sua movimentação apenas no eixo vertical. Além disso, sobre cada ponto do mesh gerado foi considerada a incidência da carga pontual através do componente do Karamba, denominado Point Load, com o intuito de simular os pesos distribuídos pela superfície.

Uma vez que este método de form-finding em princípio, não leva em consideração as propriedades dos materiais, e sim a forma geométrica, a distribuição de forças, os pontos fixados e a altura máxima da forma final, nesta simulação estrutural não foi especificado o material utilizado. Assim, através do componente do Karamba, chamado Assemble Model, os dados definidos anteriormente puderam ser reunidos de modo a gerar o modelo em elementos finitos. Finalmente, para realizar a obtenção da superfície funicular, utilizou-se o componente Large Deformation Analysis, também pertencente ao addon Karamba, sendo definida esta simulação pela altura máxima da superfície em conjunto com o número máximo de iterações a serem realizadas.

O dado de saída deste processo de obtenção da forma são linhas que formam uma casca tridimensional funicular. No entanto, em se tratando de uma análise sobre cascas maciças, necessita-se de que a geometria a ser verificada estruturalmente seja um mesh, sendo posteriormente transformado em elementos finitos de casca. Para converter estas linhas em um mesh, utilizou-se o componente do add-on Weaverbird, Mesh from Lines. A junção entre todos estes componentes do processo de form-finding utilizado é ilustrado pela Figura 2, mostrando a junção dos componentes descritos.

Com os resultados, observa-se que a forma obtida se difereda concebida por Candela em seu projeto para Los Manantiales (Figuras 3 e 4). No entanto procurou-se considerar uma altura máxima de 12,70 metros de modo que mantivesse as alturas mínimas entre o piso e a superfície de acordo com o projeto original (Figura 5).

Vale ressaltar, também, que foram considerados apoios com alguns pontos de contato para evitar que houvesse uma grande concentração de esforços nestas regiões.

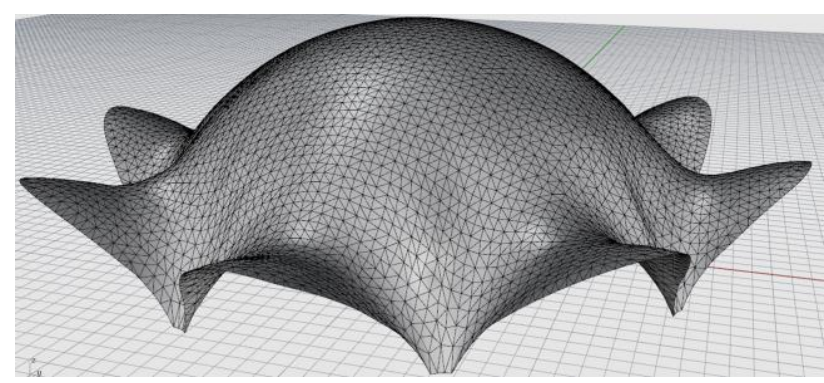

Figura 3: Perspectiva da forma obtida por form-finding. Fonte: Autores.

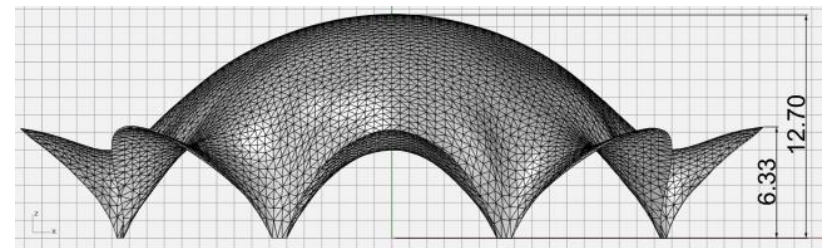

Figura 4: Vista em elevação da forma obtida por form-finding. Fonte: Autores. 


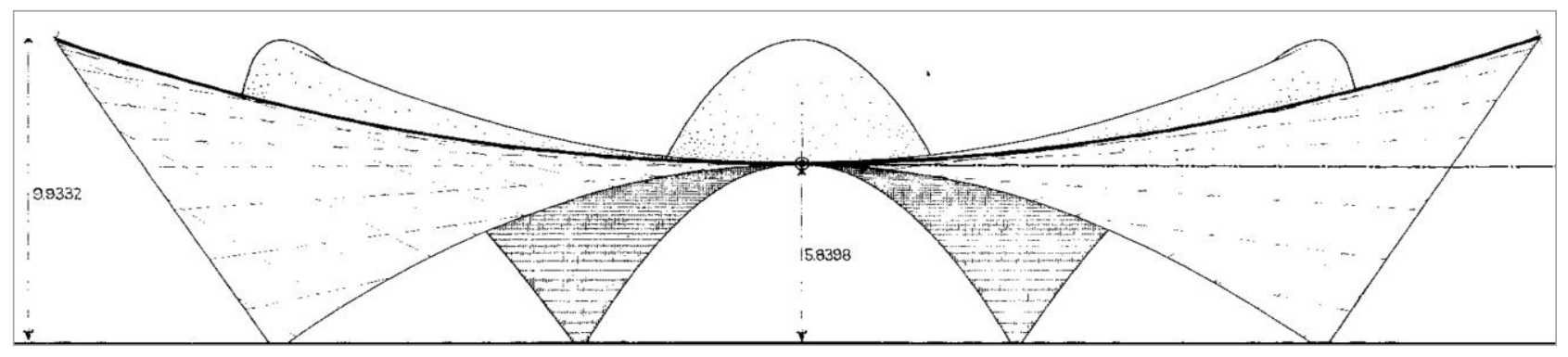

Figura 5: Corte da forma original com as alturas máximas internas de 5,83 m e 9,93 m (Faber, 1963).

\section{MODELAGEM GEOMÉTRICA DA SUPERFÍCIE DO LOS MANANTIALES}

O projeto de Candela para o restaurante em Xochimilco foi uma de suas obras de maior destaque. Nesta obra quatro seções de paraboloides hiperbólicos se reúnem em uma replicação por revolução. Este formato, apesar de ser dotado de dupla curvatura, pode ser modelado por uma linha contínua com bordas de uma seção do paraboloide formada por arestas que ligam os pontos mais altos aos mais baixos.

No processo de modelagem da forma original foi utilizado o programa Rhinoceros em conjunto com o plugin Grasshopper, utilizando-se de um processo generativo algorítmico de modo que a modelagem se tornasse mais precisa com relação às suas dimensões e posicionamentos. O processo de modelagem geométrica teve como base a proposição realizada por Alberto
Pungnale em seus estudos (Pugnale, 2009). Cada paraboloide hiperbólico pertencente à forma foi recortado por dois planos inclinados que suavizam o aspecto pontiagudo de suas extremidades e configuram as aberturas de circulação do edifício.

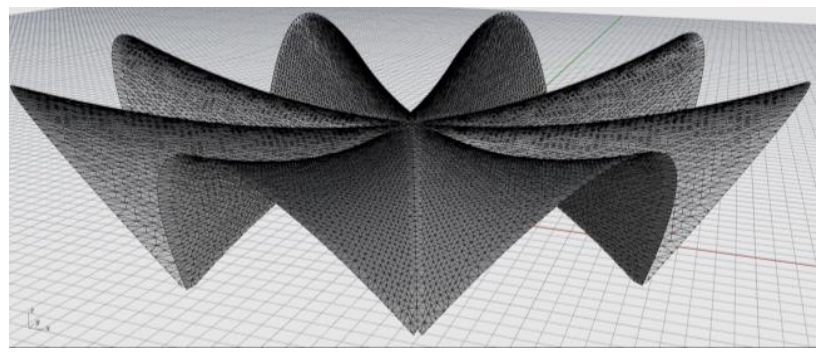

Figura 7: Perspectiva do mesh da forma do Los Manantiales. Fonte: Autores.

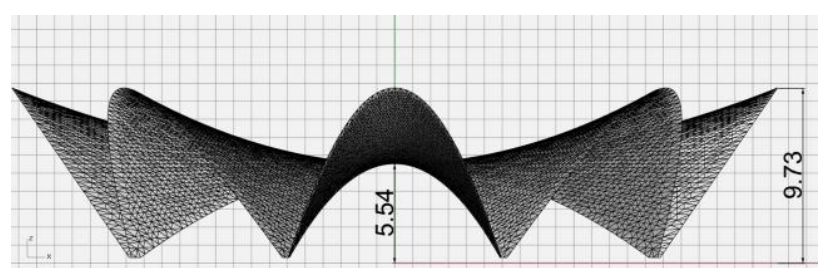

Figura 8: Elevação do mesh da forma do Los Manantiales. Fonte: Autores.

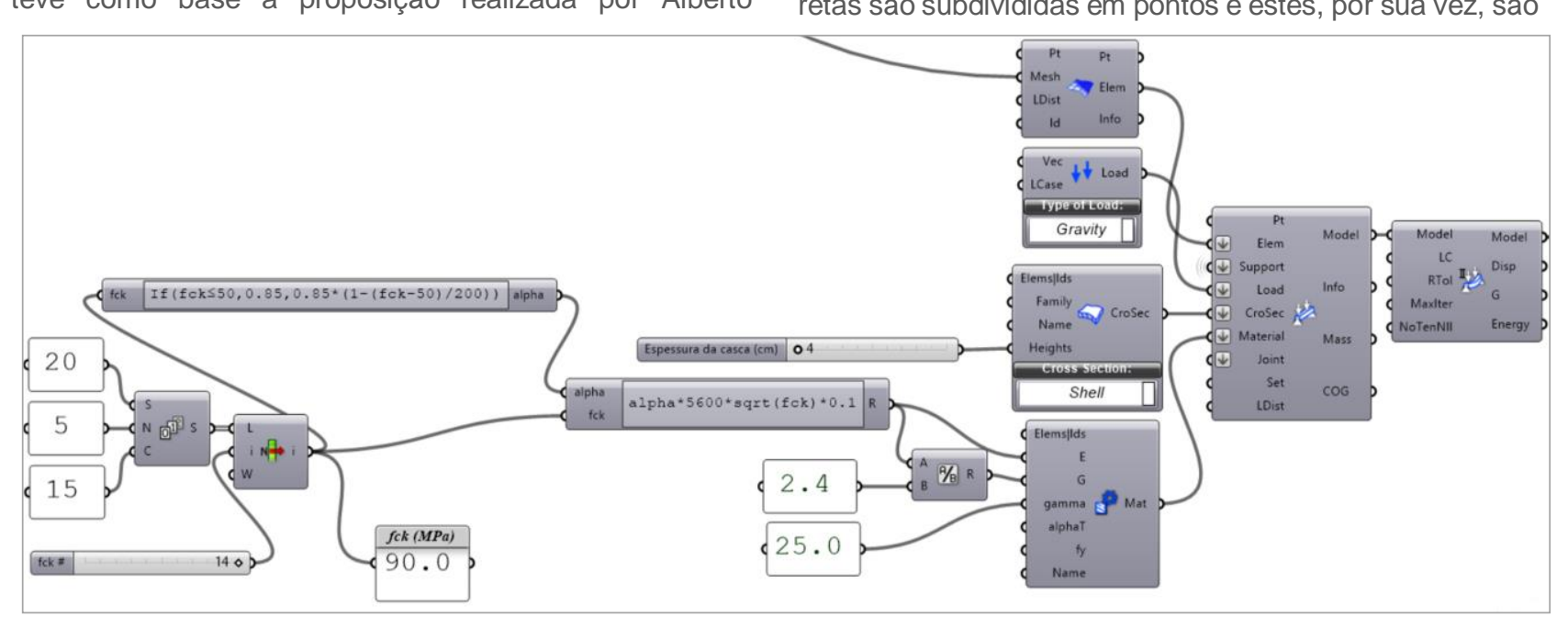

Figura 9: Trecho do código com a simulação estrutural da casca em concreto armado submetida ao peso próprio. Fonte: Autores.
A composição da forma de um recorte do paraboloide hiperbólico que serviu de base à modelagem foi obtida por traçados de linhas de forma que pudessem ser criados dois pares de retas paralelas entre si (Figura 6). Essas retas são subdivididas em pontos e estes, por sua vez, são 


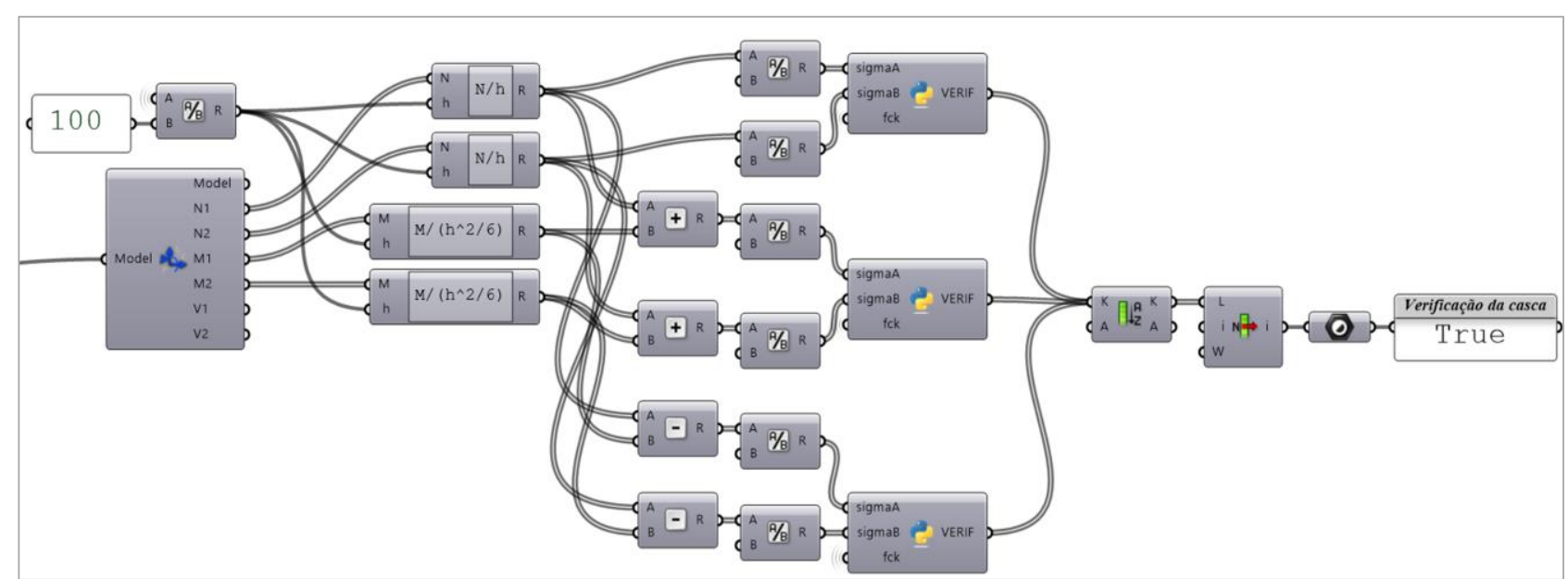

Figura 10: Trecho do código com os cálculos das tensões normais e verificação do estado de tensões na casca. Fonte: Autores.

conectados por diagonais com o objetivo de conformar ama superfície seccionada do paraboloide hiperbólico. Com a modelagem do formato do paraboloide, concebemse dois planos, intrínsecos às dimensões da forma definidas no desenho do próprio arquiteto (Faber, 1963), que recortam o paraboloide gerando a forma básica a ser revolucionada (figura 6). Esta forma resultante é replicada por revolução em torno de um eixo central a fim de conformar uma geometria muito semelhante a projetada por Candela.

Após a superfície gerada, esta foi convertida em um mesh como ilustra as figuras 7 e 8 , onde podem ser verificadas as dimensões finais, próximas às da forma original.

\section{VERIFICAÇÃO DE CASCAS EM CONCRETO ARMADO DE ACORDO COM A NBR 6118 (2014)}

Após a obtenção das formas estruturais das cascas em concreto foram realizadas sobre estas geometrias uma simulação estrutural para verificar seu comportamento em relação aos níveis de esforços, deslocamentos provenientes de deformações e o nível de suscetibilidade à flambagem.

As tensões normais nas duas direções principais sobre a superfície da casca foram calculadas em cada elemento finito do modelo a partir dos esforços normais e momentos fletores e admitindo uma largura de faixa de 1,0 m. Para isto, foi usada a equação da tensão normal proveniente de flexo-compressão (Equação 1 ). Na equação $1, h$ é a espessura da casca e $\sigma_{i}, N_{i}$ e $M_{i}$ são respectivamente, a tensão normal de flexo-compressão, o esforço normal e o momento fletor na direção $i$.

$$
\sigma_{i}=\frac{N_{i}}{h} \pm \frac{6 \cdot M_{i}}{h^{2}}
$$

Estes esforços normais e de momento fletor foram obtidos a partir de uma simulação estrutural em elementos finitos sobre as formas em investigação neste estudo. Foi considerado nestas simulações de verificação estrutural o uso de um motor de cálculo que considera os efeitos de segunda ordem para obter a resposta das formas em relação à ação do peso próprio (figura 9). Nesta modelagem pode-se observar a definição do módulo elástico e da resistência do concreto de acordo com as diretrizes da norma brasileira para projetos de estruturas em concreto armado (NBR 6118, 2014).
Na figura 10 é ilustrado o código no Grasshopper com a extração dos esforços normais e de momento fletor atuantes em cada elemento da casca, o cálculo das tensões principais segundo a equação 1 e a verificação deste nível de tensões em relação ao estado multiaxial de tensões.

Esta verificação da aceitabilidade do estado de tensões atuante foi realizada através da implementação em python script das regras para esta verificação que estão contidas no item 8.2.6 da norma NBR6118 (2014). Considerou-se que as tensões principais atuantes na casca, denominadas aqui de sigmaA e sigmaB, em conjunto com uma terceira tensão nula, perpendicular ao plano da casca, compõem o estado multiaxial de tensões, dado por $\sigma_{1} \leq \sigma_{2} \leq \sigma_{3}$. A verificação, segundo a norma, consiste em verificar se as expressões $\left(\sigma_{1} \geq-f c t d\right)$ e $\left(\sigma_{3} \leq f c d+4 \cdot \sigma_{1}\right)$ são verdadeiras, onde fctd é a tensão resistente à tração de projeto do concreto e fcd é a tensão resistente à compressão de projeto do concreto.

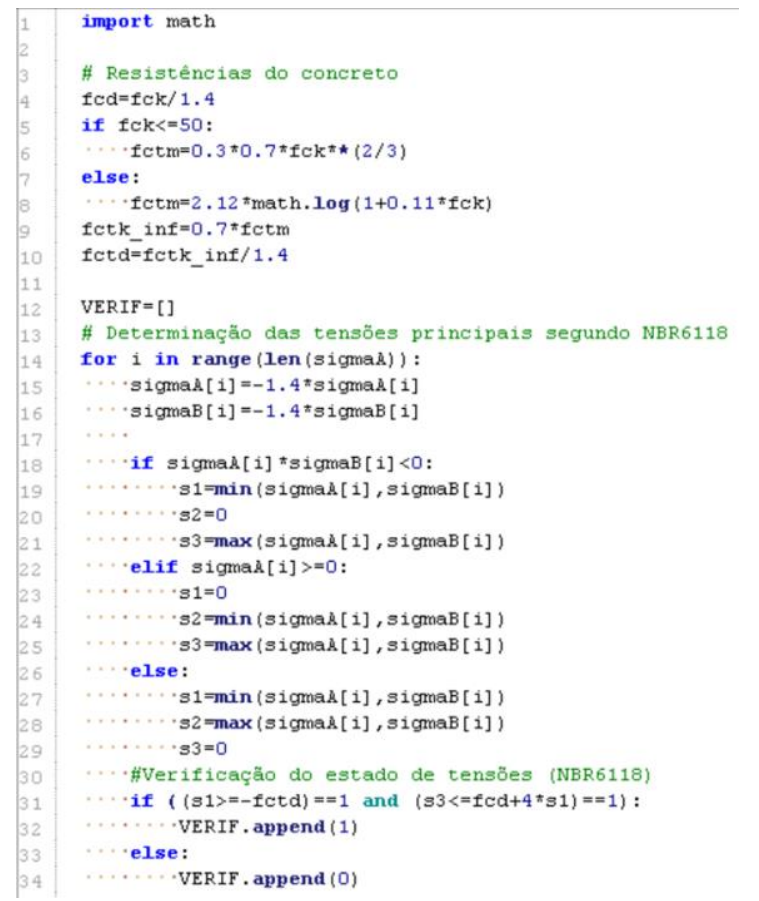

Figura 11: Script com a implementação das condicionantes para verificação do estado multiaxial de tensões no concreto. Fonte: Autores. 
Esta verificação foi implementada em Python Script, como mostra a figura 11, determinando as tensões do estado multiaxial de tensões e gerando uma lista de verificações positivas ou negativas para cada elemento do modelo.

Foram consideradas as camadas externa, interna e a que passa na altura média da espessura da casca, na qual, nesta última não se desenvolvem tensões normais que seriam oriundas do momento fletor. As respostas à verificação das três camadas foram superpostas de modo a associar cores ao mesh de elementos finitos do modelo estrutural, indicando se a região corresponde ou não aos critérios de aceitabilidade do nível de tensões normais atuantes na estrutura.

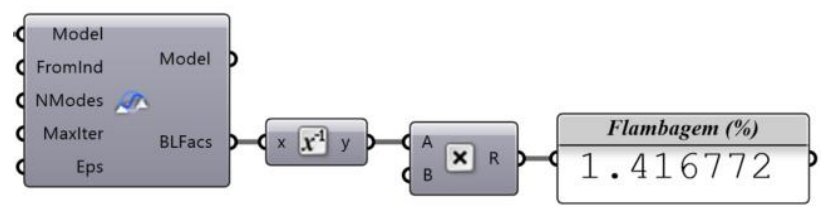

Figura 12: Verificação da casca à flambagem. Fonte: Autores.

Adicionalmente, foram considerados os cálculos dos modos de flambagem e o valor da razão entre a carga atuante em relação à carga crítica de flambagem, definido por um índice de projeto que varia de 0\% a 100\%, delimitando a suscetibilidade da estrutura quanto à flambagem. Caso seja ultrapassado os $100 \%$, a instância é suscetível à flambagem, sendo este índice obtido através do componente de cálculo de flambagem do Karamba, como ilustra a figura 12.

\section{RESULTADOS}

De acordo com Garlock et al. (2008), considerando o peso próprio da estrutura de Candela, a carga vertical da casca sobre cada um dos seus apoios é da ordem de $25.900 \mathrm{kgf}$. Considerando-se os oito apoios da estrutura, o volume aproximado de concreto do projeto original de Candela (Equação 2) pode ser obtido, então, a partir do peso específico de $2500 \mathrm{kgf} / \mathrm{m}^{3}$, dado pela norma NBR6118. Adicionalmente, os mesmos autores apresentam desenhos que informam que este volume corresponderia a uma espessura de $4,0 \mathrm{~cm}$ da casca média e uma espessura maior nas regiões dos apoios com um máximo de $12,0 \mathrm{~cm}$.

$$
V \text { con }=\frac{8 \times 25900 \mathrm{kgf}}{2500 \mathrm{kgf} / \mathrm{m}^{3}}=82,8 \mathrm{~m}^{3} \quad \text { (Eq. 2) }
$$

Inicialmente, foram verificadas as duas formas obtidas: a forma original da casca de Los Manantiales (Forma 1) e a forma obtida por form-finding (Forma 2). Para ambas se considerou inicialmente uma espessura constante de 4,0 $\mathrm{cm}$ e um valor mínimo para a resistência característica do concreto (fck), de modo que essa configuração de parâmetros resultasse em uma situação de aceitação da casca quanto resistência mecânica e da suscetibilidade à flambagem da mesma.

Na tabela 1 são informadas a espessura, a resistência característica do concreto, o volume total da casca, o deslocamento estrutural máximo e o valor que mostra a demanda à flambagem para as duas formas testadas.
Tabela 1: Características e desempenho das formas geradas

\begin{tabular}{cccccc}
\hline Forma & $\begin{array}{c}\mathrm{h} \\
(\mathrm{cm})\end{array}$ & $\begin{array}{c}\text { fck } \\
(\mathrm{MPa})\end{array}$ & $\begin{array}{c}\text { Vol } \\
\left(\mathrm{m}^{3}\right)\end{array}$ & $\begin{array}{c}\text { Desl. } \\
(\mathrm{mm})\end{array}$ & $\begin{array}{c}\text { Flamb. } \\
(\%)\end{array}$ \\
\hline 1 & 4 & 20 & 64,45 & 4,51 & 23,26 \\
2 & 4 & 90 & 59,76 & 3,64 & 3,18 \\
\hline
\end{tabular}

A figura 13 ilustra em cor azul as regiões na superfície da casca com a forma original em que o estado de tensões atende aos requisitos definidos em norma e, em vermelho, as regiões que não atendem.

A figura 14 ilustra a variação de deslocamentos ao longo da casca na forma original, apresentando um máximo de $4,51 \mathrm{~mm}$, correspondente à maior intensidade em magenta.

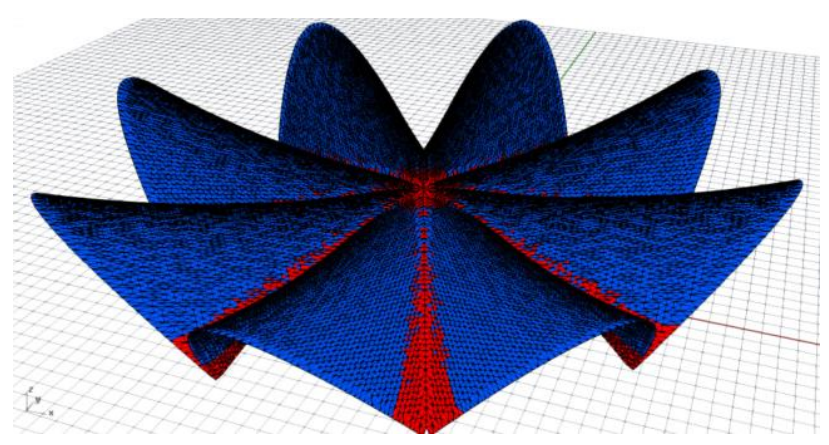

Figura 13: Critério de aceitabilidade ao estado multiaxial de tensões - Forma 1. Fonte: Autores.

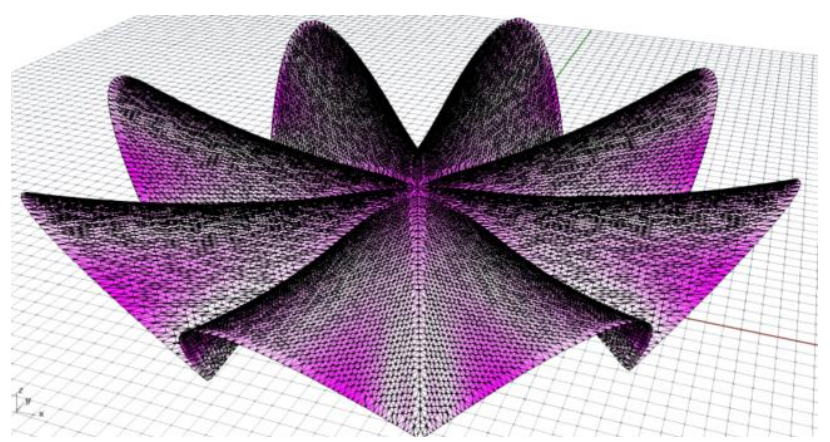

Figura 14: Padrão de deslocamentos estruturais - Forma 1. Fonte: Autores.

Analogamente, obteve-se para a casca da Forma 2 a resposta quanto ao estado de tensões na figura 15 e quanto aos deslocamentos na figura 16, sendo que este último foi de um máximo de 3,64 mm.

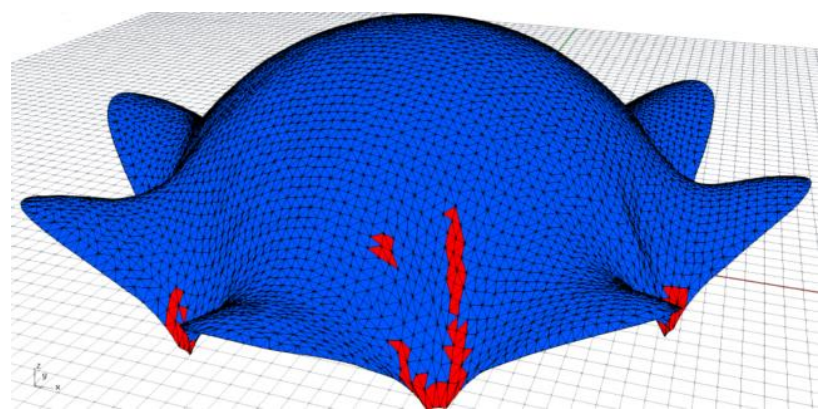

Figura 15: Critério de aceitabilidade ao estado multiaxial de tensões - Forma 2. Fonte: Autores. 


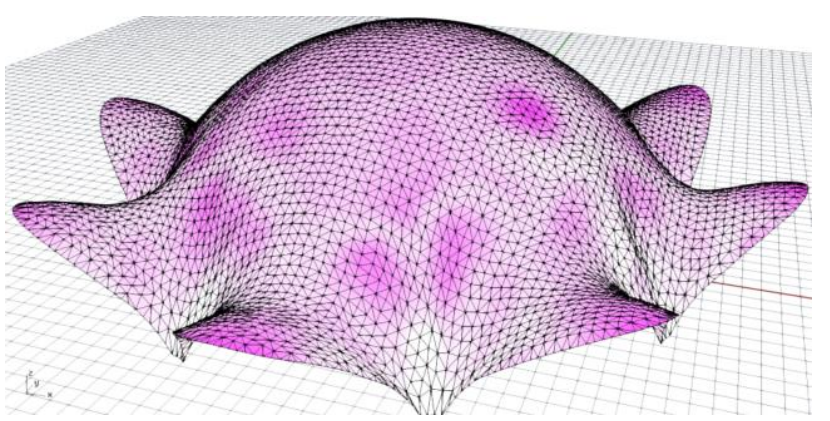

Figura 16: Padrão de deslocamentos estruturais - Forma 2. Fonte: Autores.

Observa-se nestas duas instâncias que as regiões na casca em que a condição do estado de tensões não atende aos requisitos da norma são as regiões próximas aos apoios, que irradiam para o interior da estrutura.

Considerando que a espessura de alguns dos elementos finitos não atenderam ao requisito do estado de tensões, foi realizada na sequência, uma nova simulação estrutural para verificar essa nova conformação com espessura superior (h2) nas regiões tidas como críticas. Isto foi realizado para as duas formas investigadas e os resultados estão sumarizados na tabela 2.

Tabela 2: Características/desempenho das formas modificadas

\begin{tabular}{cccrcc}
\hline Forma & $\begin{array}{c}\text { h1\&h2 } \\
(\mathrm{cm})\end{array}$ & $\begin{array}{c}\text { fck } \\
(\mathrm{MPa})\end{array}$ & $\begin{array}{c}\mathrm{V} \text { Vol } \\
\left(\mathrm{m}^{3}\right)\end{array}$ & $\begin{array}{c}\text { Desl. } \\
(\mathrm{mm})\end{array}$ & $\begin{array}{c}\text { Flamb. } \\
(\%)\end{array}$ \\
\hline $1 \mathrm{~b}$ & $4 \& 18$ & 90 & 103,25 & 1,45 & 4,26 \\
$2 \mathrm{~b}$ & $6 \& 16$ & 90 & 94,77 & 1,62 & 1,42 \\
\hline
\end{tabular}

Na tabela 2 podem ser vistos as características e índices de desempenho das cascas analisadas, com modificações nas suas espessuras e no fck.

\section{DISCUSSÃO}

Primeiramente, ficou evidente a sensibilidade e intuição de Candela frente às questões estruturais, produzindo estruturas de formas complexas mesmo sem o auxílio de ferramentas computacionais. Seus resultados, originaramse de uma série de influências e estudos prévios que permitiram o desenvolvimento de cascas em formas curvas em concreto armado.

A partir dos resultados obtidos neste estudo desenvolvido, observou-se que a estrutura de Candela demonstrou possuir um alto potencial quando se trata de eficiência estrutural frente a NBR6118.

Ainda, tanto na modelagem da forma de Candela, quanto na forma gerada pelo form-finding relativos ao Los Manantiales, nota-se a importância do fck do concreto para a obtenção de uma casca que responda às características de estabilidade estabelecidas pela norma atual. Os resultados mostram que a suscetibilidade à flambagem é diminuída quando a resistência do concreto é elevada, provavelmente devido ao aumento do módulo elástico devido ao aumento da resistência.

Observa-se que ambas as estruturas apresentaram estados de tensão críticos em seus apoios, devido à concentração dos esforços de compressão nestas regiões.
E, tratando-se especificamente da estrutura original do Los Manantiales, pode-se notar que a região crítica da união dos paraboloides coincide com a posição das vigas-arcos consideradas no projeto original (Figura 1), as quais possuem uma armação reforçada e uma espessura de concreto mais elevada nestas regiões. Para este estudo, porém, optou-se por uma variação na espessura da casca nos elementos que não atenderam os limites do estado de tensões, aumentando a espessura nestas regiões de forma a gerar um efeito semelhante ao da inserção das vigas-arco da forma original. Este aumento da espessura em determinadas regiões críticas permitiu a viabilidade das estruturas analisadas frente ao critério de tensões normais considerado segundo a norma.

O critério do estado multiaxial de tensões leva em consideração a ação conjunta das duas tensões normais principais, sem que ultrapassem os limites das tensões resistentes de projeto, tanto de tração quanto de compressão. Isto significa dizer que a armadura destas cascas estudadas e avaliadas, sob este critério, seria mínima, talvez menor do que a utilizada de fato no projeto original. Neste sentido, pode-se dizer que a taxa de armadura na estrutura projetada por esse critério poderia ser reduzida em relação ao projeto original de Candela, mesmo apresentando um acréscimo de volume de concreto de $82,3 \mathrm{~m}^{3}$ para $103,25 \mathrm{~m}^{3}$ (acréscimo de $25,4 \%$ ). Este acréscimo e a variação suave na espessura ao longo da forma original poderiam ser realizados, atualmente, com uma forma em madeira fabricada digitalmente.

Em relação à estrutura obtida por form-finding pode-se dizer que a plástica não tem o mesmo resultado estético da forma original. Observa-se que, na sua versão de espessura constante, esta casca possui uma eficiência maior em relação à forma original, consumindo menos concreto e apresentando boa rigidez. No entanto, as curvaturas da forma obtida por form-finding são complexas e localizadas em pequenas regiões de mudanças de curvaturas, que certamente geraria grandes dificuldades para sua fabricação. O que poderia ser economia em concreto, talvez se apresente como gasto excessivo em outras etapas da construção.

O uso de diversos software e tecnologias referente aos cálculos estruturais possibilitam uma melhor acessibilidade à avaliação técnica e uma maior precisão no cálculo de estruturas complexas. Deste modo, a necessidade de um empirismo físico para o desenvolvimento de novas formas é reduzida, evitando-se assim possíveis erros de projeto e permitindo, simultaneamente, a construção de estruturas complexas de maneira mais eficaz, segura e econômica.

\section{REFERÊNCIAS}

ASSOCIAÇÃO BRASILEIRA DE NORMAS TÉCNICAS (ABNT). 2014. NBR 6118: Projetos de Estruturas de Concreto procedimento. Rio de Janeiro.

Faber, C. (1963). Candela: The Shell Builder. Princeton University. Reinhold Publishing Corp.

Candela, F. (1962). Hacia una nueva filosofía de las estructuras (3 ed.). Buenos Aires: Artes Gráficas Bodoni.

Dimcic, M. S. (2011). Structural Optimization of Grid Shells based on Genetic Algorithms. Dr.-Ing Thesis on ITKE, Stuttgartt Uniersity. 
Garlock, M.; Billington, D.; and Allen, S. (2008). Building for the Future: Evaluating the Current Viability of Thin Shell Concrete Structures. Princeton University.

Pugnale, A. (2009). (Digital) Form-finding. In Tedeschi, A. (Eds.), Algorithms Aided Design: parametric strategies using grasshopper, (pp. 353-359). Brienza, Potenza: Le Penseur Publisher.
Rippmann, M. (2016). Funicular Shell Design: Geometric approaches to form-finding and fabrication of discrete funicular structures. Dr. Thesis on ETH Zurich.

Tomas, A.; Martí-Montrull, P. (2010). Optimality of Candela's concrete shells: A study of his posthumous design. Journal of the International Association for Shell and Spatial Structures, 51, 67-77. 